\title{
Atom-only theories for U(1) symmetric cavity-QED models
}

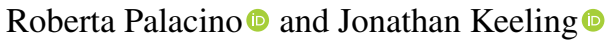 \\ SUPA, School of Physics and Astronomy, University of St Andrews, St Andrews KY16 9SS, United Kingdom
}

(Received 26 November 2020; accepted 29 June 2021; published 19 July 2021)

\begin{abstract}
We consider a generalized Dicke model with U(1) symmetry, which can undergo a transition to a superradiant state that spontaneously breaks this symmetry. By exploiting the difference in timescale between atomic and cavity dynamics, one may eliminate the cavity dynamics, providing an atom-only theory. We show that the standard Redfield theory cannot describe the transition to the superradiant state, but including higher-order corrections does recover the transition. Our work reveals how the forms of effective theories must vary for models with continuous symmetry, and provides a template to develop effective theories of more complex models.
\end{abstract}

DOI: 10.1103/PhysRevResearch.3.L032016

While phase transitions in equilibrium many-body systems have been extensively studied and are broadly understood [1,2], the critical behavior of driven-dissipative out-of-equilibrium systems [3-6] poses open questions. A central challenge in numerical exploration of such systems is the exponential growth of Hilbert space dimension with problem size. As such, any ability to reduce Hilbert space size, e.g., by a priori identifying a low-energy (slow) subspace, can be very powerful [7-10]. A widely used approach to derive a reduced model is the Redfield theory [11,12]. Previous work [13] has shown this works well for the steady states and collective modes of the Dicke model. However, as we show below, the Redfield theory can fail for models with U(1) symmetry, requiring higher-order approaches [14].

A leading platform to study driven-dissipative many-body physics is cold atoms [15] in dissipative optical cavities [16], using Raman driving [17]. This platform has been studied in a wide variety of experiments [18-30]. In its simplest form, an ensemble of $N$ atoms coupled to a cavity via Raman pumping realizes a Dicke model [17]. The Dicke model has also been used to describe a variety of other experimental systems, including nitrogen-vacancy (NV) centers in diamond [31-33], molecules [34], trapped ions [35,36], and superconducting qubits [37-43]. The Dicke model has a critical pumping strength, above which it undergoes a phase transition to a superradiant state, spontaneously breaking a $\mathbb{Z}_{2}$ symmetry [44-47]. One can also engineer more complex models, with multiple photon modes, which have U(1) [23,41,4850] or higher symmetries [51]. Furthermore, by using a degenerate (e.g., confocal) cavity, one can also explore symmetry breaking in spatially extended systems [52-54], as well as engineering exotic light-matter phases [22,26,29,55,56], tunable atomic interactions [24,28,57], and quantum memories [58-61]. In contrast to single- or few-mode systems,

Published by the American Physical Society under the terms of the Creative Commons Attribution 4.0 International license. Further distribution of this work must maintain attribution to the author(s) and the published article's title, journal citation, and DOI. continuous symmetry breaking in extended multimode systems allows one to explore the dispersion of the (complex) Goldstone modes $[4,62]$ and their contribution to critical behavior. As noted above, theoretically modeling such systems is challenging; one fruitful approach is to adiabatically eliminate fast degrees of freedom, providing an effective theory of the slow and gapless degrees of freedom. This idea motives the current work.

In this Letter, we consider a two-mode generalized Dicke model which has U(1) symmetry [50], and discuss how an effective theory can be developed. Given the motivation above, there are certain conditions required of an effective theory: It must describe the transition to a symmetry-broken state, and must correctly describe the frequencies and damping rates of the low-energy (soft modes) associated with this symmetry breaking. In the following, we first show why the standard Redfield theory fails, and then present an alternative method which succeeds. We also discuss how, for such an effective model, we can derive semiclassical equations of motion, applicable in the large $N$ limit. Importantly, the methods we present can be applied to adiabatic elimination in other problems with continuous symmetries; the U(1) Dicke model provides a proving ground for this approach.

We begin by introducing the U(1) symmetric model that we will consider, and summarizing its mean-field behavior. The model, introduced in Ref. [50], describes $N$ two-level systems-described via a collective spin degree of freedominteracting with two cavity modes. As shown in Figs. 1(a) and 1(b), this can be realized by a Raman driving scheme which couples two low-energy atomic states [17]. As shown, two transition pathways exist. Each pathway involves a different cavity mode - this is key to realizing a U(1) symmetry. Including cavity losses, we find an equation of motion for density matrix of the total system, $\rho_{t}$,

$$
\begin{gathered}
\dot{\rho}_{t}=-i\left[H, \rho_{t}\right]+\frac{\kappa}{2}(\mathcal{L}[a]+\mathcal{L}[b]), \\
H=\omega_{A} a^{\dagger} a+\omega_{B} b^{\dagger} b+\omega_{0} S^{z}+g\left[\left(a^{\dagger}+b\right) S^{-}+\text {H.c. }\right]
\end{gathered}
$$

Here, $a, b$ are the two cavity mode annihilation operators, while $\mathbf{S}$ is a collective spin of the atoms, with modulus $N / 2$. 
(a)

(b)
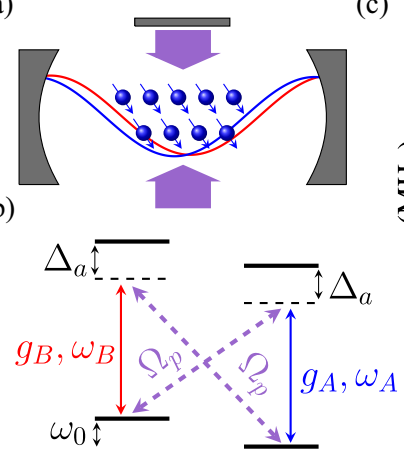

(c)

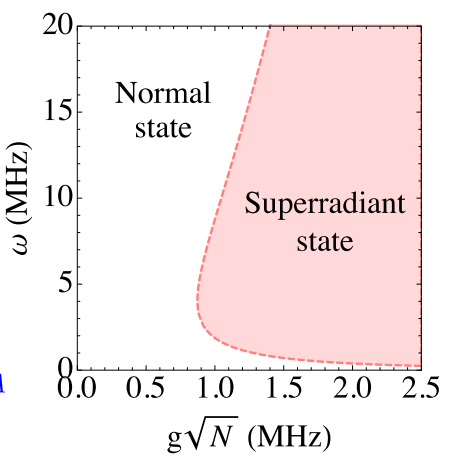

FIG. 1. (a) Experimental setup: We assume all atoms are coupled identically to cavity modes (red and blue) and pumped transverse to the cavity (amethyst). (b) Raman driving scheme. (c) Phases of the system. The pink dashed line represents the critical coupling $g_{c} \sqrt{N}$. Parameters: $\kappa=8.1 \mathrm{MHz}, \omega_{0}=47 \mathrm{kHz}, \omega_{A}=\omega_{B}=\omega$.

Cavity loss at rate $\kappa$ is described by Lindblad terms $\mathcal{L}[X]=$ $2 X \rho_{t} X^{\dagger}-\left\{X^{\dagger} X, \rho_{t}\right\}$. In the Hamiltonian, the energies $\omega_{A, B}$ describe the cost of scattering a photon from the pump into each cavity mode, while $\omega_{0}$ is the splitting of the two hyperfine atomic levels. The effective coupling between light and matter is given by $g=g_{0} \Omega_{p} / \Delta_{a}^{2}$, where $g_{0}$ is the bare atom-cavity coupling, $\Omega_{p}$ the Rabi frequency of the pump field, and $\Delta_{a}$ is the detuning between the pump and the atomic resonance [see Fig. 1(b)]. As shown in Ref. [50], this model is invariant under a transformation $\rho_{t} \rightarrow U \rho_{t} U^{\dagger}$, with $U=\exp \left[i \theta\left(S^{z}+a^{\dagger} a-\right.\right.$ $\left.\left.b^{\dagger} b\right)\right]$, which transforms $\left(a, b, S^{ \pm}\right) \rightarrow\left(a e^{i \theta}, b e^{-i \theta}, S^{ \pm} e^{\mp i \theta}\right)$. This corresponds to a $\mathrm{U}(1)$ symmetry.

For large $N$, the composite atom-cavity system is well described by semiclassical equations [63] for $\langle a\rangle,\langle b\rangle,\langle\mathbf{S}\rangle$, which show two distinct types of steady state. At small $g$, there is a normal phase with $\left\langle S^{ \pm}\right\rangle=\langle a\rangle=\langle b\rangle=0$, respecting the U(1) symmetry. At large $g$, there is a superradiant phase with a nonzero photonic field, $\langle a\rangle,\langle b\rangle \neq 0$. Thus, at a critical value $g=g_{c}$-which, for $\omega_{A}=\omega_{B}=\omega$ obeys $2 g_{c}^{2} N=$ $\omega_{0}\left(\omega^{2}+\kappa^{2} / 4\right) / \omega$ - the system undergoes a continuous phase transition to a state which spontaneously breaks the U(1) symmetry as shown in Fig. 1(c). Because of the cavity loss, these steady states are attractors of the dynamics, and the system undergoes damped relaxation towards these states.

In typical experiments, there is a separation of timescales between the atomic and cavity degrees of freedom, $\kappa \gg$ $\omega_{0}, g \sqrt{N}$. We thus next consider how to adiabatically eliminate the cavity degrees of freedom and still describe the same behavior as discussed above.

We first consider the adiabatic elimination of $\langle a\rangle,\langle b\rangle$ from the semiclassical equations given in Ref. [50]. This yields an equation of motion for $\mathbf{S}=\langle\mathbf{S}\rangle$. The resulting equation is conservative, defined by a Poisson bracket $\dot{\mathbf{S}}=\left\{\mathbf{S}, H_{\mathrm{sc}}\right\}$ where, for $\omega_{A}=\omega_{B}=\omega$, the classical Hamiltonian takes the form $H_{\mathrm{sc}}=\omega_{0} S^{z}+\frac{2 g^{2} \omega}{\omega^{2}+\kappa^{2} / 4}\left(S^{z}\right)^{2}$. While this Hamiltonian has a ground state phase transition at $g=g_{c}$ as expected, the purely conservative dynamics is in contrast with the dissipative evolution expected for an open system. Similar behavior was found for the single-mode Dicke model [13,64]. The results of Ref. [13] suggest that to recover the correct dissipative dynamics one should instead eliminate the cavity degrees of freedom in a quantum model and then derive the semiclassical limit.

To eliminate the cavity modes in a full quantum model, we use the standard Redfield approach $[11,12]$. Specifically, we take the collective spin as the system, and all other modes form the bath. The system-bath coupling in the interaction picture is $H_{I}=g\left[S^{+}(t) X(t)+S^{-}(t) X^{\dagger}(t)\right]$, where $S^{ \pm}(t)=$ $S^{ \pm} e^{ \pm i \omega_{0} t}$ and $X(t)=a(t)+b^{\dagger}(t)$. The time evolution of $X(t)$ is discussed below. Redfield theory states

$$
\dot{\rho}=-\int_{-\infty}^{t} d t^{\prime} \operatorname{Tr}_{B}\left(\left[H_{I}(t),\left[H_{I}\left(t^{\prime}\right), \rho_{t}(t)\right]\right]\right) .
$$

Evaluating this requires two-time correlations of the bath operators $X(t)$. These are found by solving Heisenberg-Langevin equations for the cavity modes, including loss, giving $\left\langle X(t) X^{\dagger}\left(t^{\prime}\right)\right\rangle=e^{-i \omega_{A}\left|t-t^{\prime}\right|-\frac{\kappa}{2}\left|t-t^{\prime}\right|}$, $\left\langle X^{\dagger}(t) X\left(t^{\prime}\right)\right\rangle=e^{-i \omega_{B}\left|t-t^{\prime}\right|-\frac{\kappa}{2}\left|t-t^{\prime}\right|}$. In the Schrödinger picture, the second-order Redfield equation (2RE) is thus

$$
\begin{aligned}
\dot{\rho}= & -i \omega_{0}\left[S^{z}, \rho\right]-2 g^{2}\left[Q_{-}\left(S^{+} S^{-} \rho-S^{-} \rho S^{+}\right)\right. \\
& \left.+Q_{+}\left(S^{-} S^{+} \rho-S^{+} \rho S^{-}\right)+\text {H.c. }\right],
\end{aligned}
$$

where $Q_{\mp}=\left[\kappa+2 i\left(\omega_{A(B)} \mp \omega_{0}\right)\right]^{-1}$.

While this equation includes dissipative effects, as we discuss next, it does not show a phase transition with increasing $g$. This is surprising, as a similar $2 \mathrm{RE}$ for the $\mathbb{Z}_{2}$ Dicke model [13] does recover its phase transition. To understand the difference here, we first rewrite this equation in Linblad form $\dot{\rho}=-i\left[H_{2 \mathrm{RE}}, \rho\right]+4 g^{2}\left(\operatorname{Re}\left[Q_{-}\right] \mathcal{L}\left[S^{-}\right]+\right.$ $\left.\operatorname{Re}\left[Q_{+}\right] \mathcal{L}\left[S^{+}\right]\right)$, where $H_{2 \mathrm{RE}}=\omega_{0} S^{z}+2 g^{2}\left(\operatorname{Im}\left[Q_{-}\right] S^{+} S^{-}+\right.$ $\operatorname{Im}\left[Q_{+}\right] S^{-} S^{+}$) commutes with $S^{z}$. The U(1) symmetry means the steady state must commute with $S^{z}$, which implies $\rho=$ $\sum_{M} P_{M}|M\rangle\langle M|$, where $S^{z}|M\rangle=M|M\rangle$. We find $P_{M}$ obeys $P_{M} / P_{M+1}=\operatorname{Re}\left[Q_{-}\right] / \operatorname{Re}\left[Q_{+}\right]$. Since this ratio is independent of $g$, no transition occurs at $g=g_{c}$. At large $N$ we find $\left\langle S^{z}\right\rangle=$ $-N / 2$ for $\operatorname{Re}\left[Q_{-}\right]>\operatorname{Re}\left[Q_{+}\right]$, or $\left\langle S^{z}\right\rangle=+N / 2$ if $\operatorname{Re}\left[Q_{-}\right]<$ $\operatorname{Re}\left[Q_{+}\right]$-i.e., the system is always in a normal or inverted state. This absence of a phase transition depends on two features of the equation. First, U(1) symmetry implies that both the effective Hamiltonian and steady-state density matrix must be diagonal in the $S^{z}$ basis - any off-diagonal term would not be invariant under the $\mathrm{U}(1)$ symmetry. As a result, the density matrix and effective Hamiltonian always commute-a statement that will always be true for U(1) symmetric models. This means that the form of the steady state must be determined by the dissipative terms. Second, the dissipative terms in the Redfield equation are all proportional to $g^{2}$, so no $g$ dependence occurs in their ratio. This statement is not generic, so we next consider how contributions of higher order in $g$ change the equation.

A systematic method to derive higher-order density matrix equations was introduced by Müller and Stace [14], making use of the Keldysh diagrammatic perturbation theory. This technique allows one to take into account all contributions at each order while avoiding double counting. We write the density matrix equation in the form $\dot{\rho}=\mathcal{D}_{0} \rho+\mathcal{D}_{2} \rho+\mathcal{D}_{4} \rho$, where $\mathcal{D}_{0} \rho+\mathcal{D}_{2} \rho$ is given in Eq. (4), and $\mathcal{D}_{4} \rho$ is fourth order in $H_{I}$. Crucially, the diagrammatic expansion ensures the terms in $\mathcal{D}_{4} \rho$ are not separable-i.e., they correspond to 

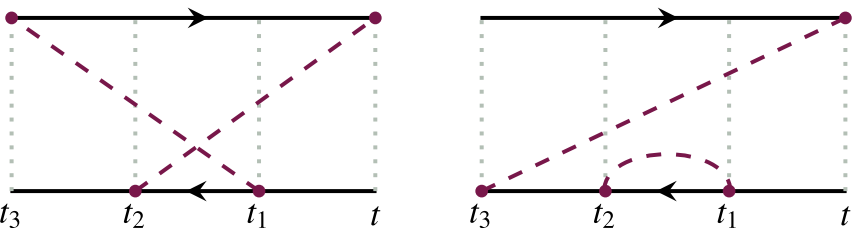

FIG. 2. Example diagrams at fourth order. The solid black lines are periods of free evolution of the system, interrupted by the action of the interaction Hamiltonian at times $t, t_{1}, t_{2}, t_{3}$ (purple dots). These vertices are connected by purple dashed lines representing the cavity mode correlation functions.

genuine fourth-order processes, not products of second-order terms.

Figure 2 shows two examples of fourth-order diagrams. As described in Ref. [14], the solid horizontal lines indicate the branches of the Keldysh contour. The system undergoes free evolution along these branches, interrupted by the action of $H_{I}$ at times $t>t_{1}>t_{2}>t_{3}$ (purple dots). Points on the lower branch correspond to operators to the left of the density matrix, while those on the upper branch are to the right. Because the bath is quadratic, expectations of bath operators factorize into pairwise correlations (purple dashed lines). The form of $H_{I}$ means each dashed line must connect opposite $S^{ \pm}$ operators. Following these rules, after integrating over $t_{1}, t_{2}, t_{3}$ the diagrams in Fig. 2 correspond to

$$
\begin{aligned}
4 g^{4} & {\left[\left|Q_{-}\right|^{2} \kappa^{-1} S^{-} S^{-} \rho S^{+} S^{+}+\left|Q_{+}\right|^{2} \kappa^{-1} S^{+} S^{+} \rho S^{-} S^{-}\right.} \\
& +Q_{-} Q_{+}^{*} Q_{\Delta} S^{+} S^{-} \rho S^{-} S^{+}+Q_{-}^{*} Q_{+} Q_{\Delta}^{*} S^{-} S^{+} \rho S^{+} S^{-} \\
& -Q_{+}^{2} Q_{\Sigma} S^{+} S^{-} S^{+} \rho S^{-}-Q_{-}^{2} Q_{\Sigma} S^{-} S^{+} S^{-} \rho S^{+} \\
& \left.-Q_{-}^{3} S^{+} S^{-} S^{-} \rho S^{+}-Q_{+}^{3} S^{-} S^{+} S^{+} \rho S^{-}\right],
\end{aligned}
$$

with $Q_{\Delta}=\left[\kappa+i\left(\omega_{A}-\omega_{B}-2 \omega_{0}\right)\right]^{-1}$, and $Q_{\Sigma}=$ $\left[\kappa+i\left(\omega_{A}+\omega_{B}\right)\right]^{-1}$. Overall, at fourth order, there are 32 diagrams. Considering the patterns of $S^{ \pm}$operators, each diagram contributes four terms. These are written in full in the Supplemental Material (SM) [65].

The resulting equation is not of Lindblad form [66] and so does not necessarily preserve positivity-this is shown in the SM [65], where we diagonalize the Lindblad-Kossakowski matrix. As discussed in many other contexts, such nonpositive equations can nonetheless predict correct behavior [13,6771]. In second-order theories, a Lindblad form equation is known to arise from secularizating the Redfield equation [72]-i.e., deleting terms which are time dependent in the interaction picture. Since our equation is U(1) symmetric, it already has a secularized form.

While U(1) symmetry still means the density matrix is diagonal in the $S^{z}$ basis, the presence of the fourth-order contribution gives a nontrivial dependence on $g$. In particular, as shown in Fig. 3(a), the steady state of this fourth-order Keldysh-Redfield equation (4KRE) shows a transition to a superradiant state. As $N$ increases, the results converge to the mean-field predictions of the full atom-cavity model, with a discontinuity in $\left\langle S^{z}\right\rangle$ at $g=g_{c}$. Such a change in the spin state could be directly detected by spin-resolved imaging, as used in Ref. [29], or by associated changes in the cavity photon occupation [47].

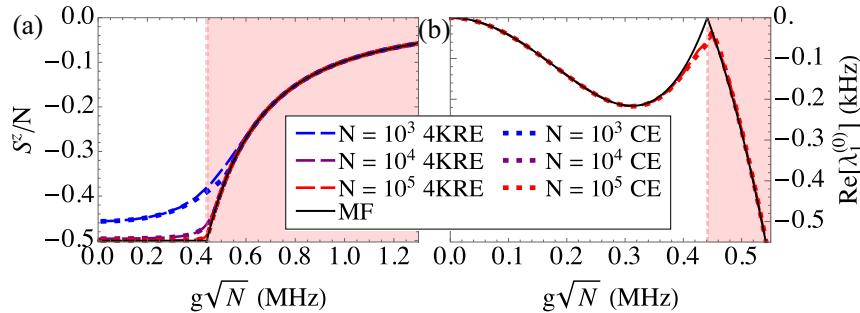

FIG. 3. (a) Steady-state $\left\langle S^{z}\right\rangle$ evaluated from $4 \mathrm{KRE}$ (dashed lines) for three system sizes $N=10^{3}, 10^{4}, 10^{5}$. The results are compared to the cumulant equations (dotted lines), and mean-field theory of the atom-cavity equations (solid black line). (b) The first nonzero eigenvalue of the $k=0$ sector of $\mathcal{L}$ for $N=10^{5}$ (dashed line), the linearized $\mathrm{CE}$ (dotted line) for $N=10^{5}$, and the mean-field atom-cavity equations (black solid line). These eigenvalues describe relaxation towards the stationary state. The pink region indicates the superradiant phase, $g>g_{c} \sqrt{N}=0.44 \mathrm{MHz}$ for the set of parameters used: $\kappa=8.1 \mathrm{MHz}, \omega_{0}=47 \mathrm{kHz}, \omega_{A}=\omega_{B}=5 \mathrm{MHz}$.

We now consider whether we can use the $4 \mathrm{KRE}$ to derive semiclassical equations which capture the dissipative phase transition. A naive approach to this is to assume that terms $\left\langle S^{\alpha} S^{\beta} S^{\gamma}\right\rangle$ can be factorized. Applying such an approach directly to $4 \mathrm{KRE}$ fails, in the sense that after factorization, this yields equations for which $\left\langle S^{x, y}\right\rangle=0,\left\langle S^{z}\right\rangle=-N / 2$ is always a stable solution, in contrast to the clear instability seen in the full quantum solution. The structure of the full quantum solution (i.e., a density matrix diagonal in the $S^{z}$ basis) suggests the origin of this failure: while $U(1)$ symmetry ensures $\left\langle S^{x, y}\right\rangle$ is zero for the full solution, products such as $\left\langle\left(S^{x}\right)^{2}\right\rangle$ can be nonzero. The diagonal structure of the steady-state density matrix suggests a better way to approach a semiclassical limit is by considering the Gaussian form of the probabilities $P_{M}$ (see SM [65]), and characterizing $P_{M}$ by its first two moments. This yields a form of cumulant equation (CE), as used elsewhere $[63,73]$. Considering coupled equations for $\left\langle S^{z} S^{z}\right\rangle,\left\langle S^{z}\right\rangle$ (given in SM [65]), one finds results [dotted lines in Fig. 3(a)] that match $4 \mathrm{KRE}$ well for a range of $N$. At $N \rightarrow \infty, P_{M}$ is sharply peaked so $\left\langle S^{z} S^{z}\right\rangle \rightarrow\left\langle S^{z}\right\rangle^{2}$, giving a single equation for $\left\langle S^{z}\right\rangle$,

$$
\partial_{t}\left\langle S^{z}\right\rangle=\left[-2 g^{2} \eta+2 g^{4} \zeta\left\langle S^{z}\right\rangle\right]\left[S^{2}-\left\langle S^{z}\right\rangle^{2}\right],
$$

where $\eta=2 \operatorname{Re}\left[Q_{-}-Q_{+}\right]$and $\zeta=8 \operatorname{Re}\left[\left(Q_{-}+Q_{+}\right)^{2} Q_{\Sigma}\right]-$ $16 \kappa^{-1}\left[\operatorname{Re}\left(Q_{-}\right)^{2}+\operatorname{Re}\left(Q_{+}\right)^{2}\right]$.

To explore whether the atom-only equations we have derived correctly capture the slow dynamics, we next consider the Liouvillian spectrum. Due to the U(1) symmetry, the quantum dynamics decouples into sectors labeled by index $k$, $\rho=\sum_{k=-S}^{S} \sum_{M=-S-\min (k, 0)}^{S-\min (k, 0)} R_{M}^{(k)}|M\rangle\langle M+k|$. The equations for each $k$ are independent: $\dot{R}_{M}^{(k)}=L_{M, M^{\prime}}^{(k)} R_{M^{\prime}}^{(k)}$. The matrices $L^{(k)}$ in each sector can be numerically diagonalized to obtain eigenvalues $\lambda_{i}^{(k)}$. The real part of these eigenvalues describe the relaxation rate toward the steady state. For our fourth-order approach, the matrices $L^{(k)}$ take a simple structure-only terms with $M^{\prime}=M, M \pm 1, M \pm 2$ are nonzero, yielding a pentadiagonal matrix [65]. Together, the separation into sectors and this banded structure mean we can numerically find the eigenspectrum for relatively large $N$. 

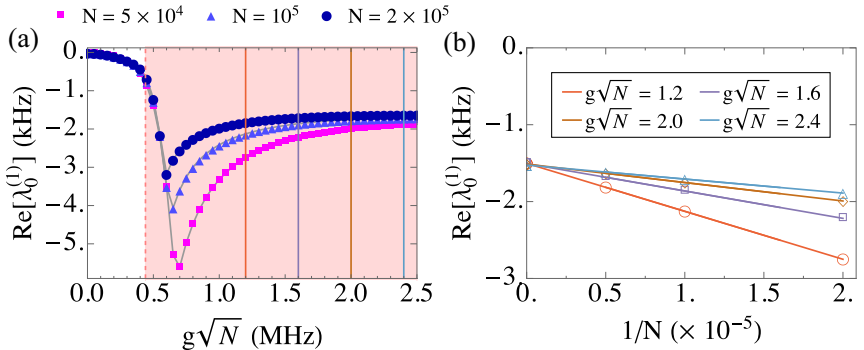

FIG. 4. Liouvillian gap in the $|k|=1$ sector. (a) Gap vs $g \sqrt{N}$. The colored vertical lines indicate $g \sqrt{N}$ values above threshold used to evaluate the trend of the gap closure with the system size shown in (b). This is computed for $N$ in the range $\left[5 \times 10^{4}-2 \times 10^{5}\right]$. The intercept shows the extrapolated gap as $N \rightarrow \infty$. All parameters as for Fig. 3.

The $k=0$ sector describes the dynamics of the populations (in the $S^{z}$ basis). As such, the eigenvalues in this sector provide information about the evolution of $\left\langle S^{z}\right\rangle$, and in particular, the damping rate towards steady state. The first nonzero eigenvalue [74] in the $k=0$ sector, $\lambda_{1}^{(0)}$, is shown in Fig. 3(b). We can also compare this eigenvalue to the decay rate found by linearizing the semiclassical Eq. (6),

$$
\lambda_{\mathrm{MF}}=4 g^{2} \eta\left\langle S^{z}\right\rangle_{\mathrm{ss}}+2 g^{4} \zeta\left(S^{2}-3\left\langle S^{z}\right\rangle_{\mathrm{ss}}^{2}\right),
$$

shown as the black solid line in Fig. 3(b).

While the first nonzero eigenvalue for $k=0$ tells us about relaxation to the steady state, it does not describe the slowest dynamics of this $U(1)$ symmetric system-i.e., the smallest nonzero eigenvalue, also known as the Liouvillian gap. As explained in Refs. [62], when an open system spontaneously breaks a symmetry, the Liouvillian gap should vanish as $N \rightarrow \infty$ throughout the symmetry-broken phase. This occurs because spontaneous symmetry breaking means more than one steady state is possible; since any mixture of two steadystate density matrices is also a steady state, an extra zero mode must arise. When the spontaneously broken symmetry is continuous, this also relates to the Goldstone mode.

For our model, the gapless mode is associated with how the U(1) symmetry is broken. As such, it must involve terms which are off diagonal in the $S^{z}$ basis-specifically terms which correspond to the long-time evolution of $\left\langle S^{+}(t) S^{-}(0)\right\rangle$. This means we should consider the smallest eigenvalue (by real part) of the $|k|=1$ sector, $\lambda_{0}^{(1)}$. This eigenvalue is shown in Fig. 4(a), as a function of coupling $g$. One may see that the gap reduces with increasing $N$. However, a detailed analysis of the Liouvillian gap as a function of system size [Fig. 4(b)] shows a nonzero gap remains at $N \rightarrow \infty$. Specif- ically, considering $g>g_{c}$, we see this eigenvalue matches well to $\lambda_{0}^{(1)}=A+B / N$, with a finite intercept $A$. As noted above, the 4KRE is not of Lindblad form (i.e., is not completely positive), and it is possible this may be associated with the nonvanishing Liouvillian gap. However, the equation is Hermitian, trace-preserving, respects the $\mathrm{U}(1)$ symmetry, and predicts the correct steady states. Moreover, other examples of nonpositive Redfield equations do show the expected vanishing Liouvillian gap, as shown in the SM [65]. We note that the behavior we observe in the 4KRE-a phase transition without a vanishing Liouvillian gap, and thus without symmetry breaking - is behavior discussed by Ref. [75] as "spectral collapse" in the context of lasing models with dephasing.

In conclusion, we have shown that constructing an atomonly effective theory for a model with U(1) symmetry presents surprising challenges. We have shown why the standard second-order Redfield theory fails to describe the superradiant transition, and we have shown how this can be rectified by the fourth-order terms of a diagrammatic expansion. We see this correctly describes the steady state and relaxation to that state in the $k=0$ sector. This result reveals how apparent failures of the Redfield theory for continuous symmetry can be addressed by higher-order approaches. Moreover, we may see how a semiclassical approximation becomes valid as $N \rightarrow \infty$, through the emergence of an increasingly sharp distribution-such an approach may provide alternate ways to understand models where it is found that the semiclassical (mean-field) approximation fails [76,77]. When considering spin coherences (i.e., the $|k|=1$ sector), we surprisingly find that the 4KRE does not predict a vanishing Liouvillian gap in the symmetry-broken state. Moreover, we note that the 4KRE provides a Hermitian, trace-preserving, and secularized density matrix equation which is nevertheless not of Lindblad form. A key task for future work is to extend the methods developed here to a full multimode (e.g., confocal cavity) and spatially extended system. This would provide a powerful tool to theoretically explore nonequilibrium phase transitions in these driven-dissipative multimode systems.

The research data supporting this publication can be accessed at [78]

We are grateful for helpful discussions with A. Daley and F. Damanet. R.P. wishes to thank G. Baio for stimulating discussions. We are grateful to B. Lev for comments on an earlier version of the manuscript. R.P. was supported by the EPSRC Scottish Doctoral Training Centre in Condensed Matter Physics (CM-CDT), Grant No. EP/L015110/1.
[1] L. Landau and E. Lifshitz, Statistical Physics, Course of Theoretical Physics Vol. 5 (Butterworth-Heinemann, Oxford, U.K., 1980).

[2] P. Chaikin and T. Lubensky, Principles of Condensed Matter Physics (Cambridge University Press, Cambridge, UK, 1995).

[3] S. Diehl, A. Micheli, A. Kantian, B. Kraus, H. Büchler, and P. Zoller, Quantum states and phases in driven open quantum systems with cold atoms, Nat. Phys. 4, 878 (2008).
[4] I. Carusotto and C. Ciuti, Quantum fluids of light, Rev. Mod. Phys. 85, 299 (2013).

[5] K. Le Hur, L. Henriet, A. Petrescu, K. Plekhanov, G. Roux, and M. Schiró, Many-body quantum electrodynamics networks: Non-equilibrium condensed matter physics with light, C. R. Acad. Sci. 17, 808 (2016).

[6] D. E. Chang, J. S. Douglas, A. González-Tudela, C.-L. Hung, and H. J. Kimble, Colloquium: Quantum matter built from 
nanoscopic lattices of atoms and photons, Rev. Mod. Phys. 90, 031002 (2018).

[7] E. M. Kessler, Generalized Schrieffer-Wolff formalism for dissipative systems, Phys. Rev. A 86, 012126 (2012).

[8] B. Sciolla, D. Poletti, and C. Kollath, Two-Time Correlations Probing the Dynamics of Dissipative Many-Body Quantum Systems: Aging and Fast Relaxation, Phys. Rev. Lett. 114, 170401 (2015).

[9] Z. Lenarčič, F. Lange, and A. Rosch, Perturbative approach to weakly driven many-particle systems in the presence of approximate conservation laws, Phys. Rev. B 97, 024302 (2018).

[10] I. Saideh, D. Finkelstein-Shapiro, C. Noûs, T. Pullerits, and A. Keller, Projection-based adiabatic elimination of bipartite open quantum systems, Phys. Rev. A 102, 032212 (2020).

[11] A. G. Redfield, On the theory of relaxation processes, IBM J. Res. Dev. 1, 19 (1957).

[12] H.-P. Breuer and F. Petruccione, The Theory of Open Quantum Systems (Oxford University Press, Oxford, U.K., 2002).

[13] F. Damanet, A. J. Daley, and J. Keeling, Atom-only descriptions of the driven-dissipative Dicke model, Phys. Rev. A 99, 033845 (2019).

[14] C. Müller and T. M. Stace, Deriving Lindblad master equations with Keldysh diagrams: Correlated gain and loss in higher order perturbation theory, Phys. Rev. A 95, 013847 (2017).

[15] I. Bloch, J. Dalibard, and W. Zwerger, Many-body physics with ultracold gases, Rev. Mod. Phys. 80, 885 (2008).

[16] P. Domokos and H. Ritsch, Mechanical effects of light in optical resonators, J. Opt. Soc. Am. B 20, 1098 (2003).

[17] F. Dimer, B. Estienne, A. S. Parkins, and H. J. Carmichael, Proposed realization of the Dicke-model quantum phase transition in an optical cavity QED system, Phys. Rev. A 75, 013804 (2007).

[18] K. Baumann, C. Guerlin, F. Brennecke, and T. Esslinger, Dicke quantum phase transition with a superfluid gas in an optical cavity, Nature (London) 464, 1301 (2010).

[19] H. Keßler, J. Klinder, M. Wolke, and A. Hemmerich, Steering Matter Wave Superradiance with an Ultranarrow-Band Optical Cavity, Phys. Rev. Lett. 113, 070404 (2014).

[20] J. Klinder, H. Keßler, M. R. Bakhtiari, M. Thorwart, and A. Hemmerich, Observation of a superradiant Mott insulator in the Dicke-Hubbard model, Phys. Rev. Lett. 115, 230403 (2015).

[21] R. Landig, L. Hruby, N. Dogra, M. Landini, R. Mottl, T. Donner, and T. Esslinger, Quantum phases from competing short- and long-range interactions in an optical lattice, Nature (London) 532, 476 (2016).

[22] A. J. Kollár, A. T. Papageorge, V. D. Vaidya, Y. Guo, J. Keeling, and B. L. Lev, Supermode-density-wave-polariton condensation with a Bose-Einstein condensate in a multimode cavity, Nat. Commun. 8, 14386 (2017).

[23] J. Léonard, A. Morales, P. Zupancic, T. Esslinger, and T. Donner, Supersolid formation in a quantum gas breaking a continuous translational symmetry, Nature (London) 543, 87 (2017).

[24] V. D. Vaidya, Y. Guo, R. M. Kroeze, K. E. Ballantine, A. J. Kollár, J. Keeling, and B. L. Lev, Tunable-Range, PhotonMediated Atomic Interactions in Multimode Cavity QED, Physical Review X 8, 011002 (2018).
[25] M. Landini, N. Dogra, K. Kroeger, L. Hruby, T. Donner, and T. Esslinger, Formation of a Spin Texture in a Quantum Gas Coupled to a Cavity, Phys. Rev. Lett. 120, 223602 (2018).

[26] R. M. Kroeze, Y. Guo, V. D. Vaidya, J. Keeling, and B. L. Lev, Spinor Self-Ordering of a Quantum Gas in a Cavity, Phys. Rev. Lett. 121, 163601 (2018).

[27] Z. Zhang, C. H. Lee, R. Kumar, K. J. Arnold, S. J. Masson, A. L. Grimsmo, A. S. Parkins, and M. D. Barrett, Dicke-model simulation via cavity-assisted Raman transitions, Phys. Rev. A 97, 043858 (2018).

[28] Y. Guo, R. M. Kroeze, V. D. Vaidya, J. Keeling, and B. L. Lev, Sign-Changing Photon-Mediated Atom Interactions in Multimode Cavity Quantum Electrodynamics, Phys. Rev. Lett. 122, 193601 (2019).

[29] R. M. Kroeze, Y. Guo, and B. L. Lev, Dynamical Spin-Orbit Coupling of a Quantum Gas, Phys. Rev. Lett. 123, 160404 (2019).

[30] E. J. Davis, G. Bentsen, L. Homeier, T. Li, and M. H. SchleierSmith, Photon-Mediated Spin-Exchange Dynamics of Spin-1 Atoms, Phys. Rev. Lett. 122, 010405 (2019).

[31] L. Zou, D. Marcos, S. Diehl, S. Putz, J. Schmiedmayer, J. Majer, and P. Rabl, Implementation of the Dicke Lattice Model in Hybrid Quantum System Arrays, Phys. Rev. Lett. 113, 023603 (2014).

[32] S. Putz, D. O. Krimer, R. Amsuess, A. Valookaran, T. Noebauer, J. Schmiedmayer, S. Rotter, and J. Majer, Protecting a spin ensemble against decoherence in the strong-coupling regime of cavity QED, Nat. Phys. 10, 720 (2014).

[33] A. Angerer, K. Streltsov, T. Astner, S. Putz, H. Sumiya, S. Onoda, J. Isoya, W. J. Munro, K. Nemoto, J. Schmiedmayer et al., Superradiant emission from colour centres in diamond, Nat. Phys. 14, 1168 (2018).

[34] P. Rabl, D. DeMille, J. M. Doyle, M. D. Lukin, R. Schoelkopf, and P. Zoller, Hybrid Quantum Processors: Molecular Ensembles as Quantum Memory for Solid State Circuits, Phys. Rev. Lett. 97, 033003 (2006).

[35] S. Genway, W. Li, C. Ates, B. P. Lanyon, and I. Lesanovsky, Generalized Dicke Nonequilibrium Dynamics in Trapped Ions, Phys. Rev. Lett. 112, 023603 (2014).

[36] A. Safavi-Naini, R. Lewis-Swan, J. G. Bohnet, M. Gärttner, K. A. Gilmore, J. E. Jordan, J. Cohn, J. K. Freericks, A. M. Rey, and J. J. Bollinger, Verification of a Many-Ion Simulator of the Dicke Model Through Slow Quenches Across a Phase Transition, Phys. Rev. Lett. 121, 040503 (2018).

[37] P. Nataf and C. Ciuti, No-go theorem for superradiant quantum phase transitions in cavity QED and counter-example in circuit QED, Nat. Commun. 1, 72 (2010).

[38] O. Viehmann, J. von Delft, and F. Marquardt, Superradiant Phase Transitions and the Standard Description of Circuit QED, Phys. Rev. Lett. 107, 113602 (2011).

[39] P. Nataf, A. Baksic, and C. Ciuti, Double symmetry breaking and two-dimensional quantum phase diagram in spin-boson systems, Phys. Rev. A 86, 013832 (2012).

[40] A. A. Houck, H. E. Türeci, and J. Koch, On-chip quantum simulation with superconducting circuits, Nat. Phys. 8, 292 (2012).

[41] A. Baksic and C. Ciuti, Controlling Discrete and Continuous Symmetries in Superradiant Phase Transitions with Circuit QED Systems, Phys. Rev. Lett. 112, 173601 (2014). 
[42] N. Lambert, Y. Matsuzaki, K. Kakuyanagi, N. Ishida, S. Saito, and F. Nori, Superradiance with an ensemble of superconducting flux qubits, Phys. Rev. B 94, 224510 (2016).

[43] M. Bamba, K. Inomata, and Y. Nakamura, Superradiant Phase Transition in a Superconducting Circuit in Thermal Equilibrium, Phys. Rev. Lett. 117, 173601 (2016).

[44] K. Hepp and E. H. Lieb, Equilibrium statistical mechanics of matter interacting with the quantized radiation field, Phys. Rev. A 8, 2517 (1973).

[45] Y. K. Wang and F. T. Hioe, Phase transition in the Dicke model of superradiance, Phys. Rev. A 7, 831 (1973).

[46] B. M. Garraway, The Dicke model in quantum optics: Dicke model revisited, Philos. Trans. R. Soc. London, Ser. A 369, 1137 (2011).

[47] P. Kirton, M. M. Roses, J. Keeling, and E. G. Dalla Torre, Introduction to the Dicke model: From equilibrium to nonequilibrium, and vice versa, Adv. Quantum Technol. 2, 1800043 (2019).

[48] J. Fan, Z. Yang, Y. Zhang, J. Ma, G. Chen, and S. Jia, Hidden continuous symmetry and Nambu-Goldstone mode in a twomode Dicke model, Phys. Rev. A 89, 023812 (2014).

[49] J. Léonard, A. Morales, P. Zupancic, T. Donner, and T. Esslinger, Monitoring and manipulating Higgs and Goldstone modes in a supersolid quantum gas, Science 358, 1415 (2017).

[50] R. I. Moodie, K. E. Ballantine, and J. Keeling, Generalized classes of continuous symmetries in two-mode Dicke models, Phys. Rev. A 97, 033802 (2018).

[51] E. I. R. Chiacchio and A. Nunnenkamp, Emergence of continuous rotational symmetries in ultracold atoms coupled to optical cavities, Phys. Rev. A 98, 023617 (2018).

[52] S. Gopalakrishnan, B. L. Lev, and P. M. Goldbart, Emergent crystallinity and frustration with Bose-Einstein condensates in multimode cavities, Nat. Phys. 5, 845 (2009).

[53] S. Gopalakrishnan, B. L. Lev, and P. M. Goldbart, Atom-light crystallization of Bose-Einstein condensates in multimode cavities: Nonequilibrium classical and quantum phase transitions, emergent lattices, supersolidity, and frustration, Phys. Rev. A 82, 043612 (2010).

[54] G. Labeyrie, E. Tesio, P. M. Gomes, G. L. Oppo, W. J. Firth, G. R. M. Robb, A. S. Arnold, R. Kaiser, and T. Ackemann, Optomechanical self-structuring in a cold atomic gas, Nat. Photonics 8, 321 (2014).

[55] K. E. Ballantine, B. L. Lev, and J. Keeling, Meissner-like Effect for a Synthetic Gauge Field in Multimode Cavity QED, Phys. Rev. Lett. 118, 045302 (2017).

[56] C. Rylands, Y. Guo, B. L. Lev, J. Keeling, and V. Galitski, Photon-Mediated Peierls Transition of a 1D Gas in a Multimode Optical Cavity, Phys. Rev. Lett. 125, 010404 (2020).

[57] Y. Guo, V. D. Vaidya, R. M. Kroeze, R. A. Lunney, B. L. Lev, and J. Keeling, Emergent and broken symmetries of atomic self-organization arising from Gouy phase shifts in multimode cavity QED, Phys. Rev. A 99, 53818 (2019).

[58] S. Gopalakrishnan, B. L. Lev, and P. M. Goldbart, Exploring models of associative memory via cavity quantum electrodynamics, Philos. Mag. 92, 353 (2012).

[59] E. Fiorelli, M. Marcuzzi, P. Rotondo, F. Carollo, and I. Lesanovsky, Signatures of Associative Memory Behavior in a Multimode Dicke Model, Phys. Rev. Lett. 125, 070604 (2020).
[60] B. P. Marsh, Y. Guo, R. M. Kroeze, S. Gopalakrishnan, S. Ganguli, J. Keeling, and B. L. Lev, Enhancing associative memory recall and storage capacity using confocal cavity QED, Phys. Rev. X 11, 021048 (2021).

[61] F. Carollo and I. Lesanovsky, Exactness of Mean-Field Equations for Open Dicke Models with an Application to Pattern Retrieval Dynamics, Phys. Rev. Lett. 126, 230601 (2021).

[62] F. Minganti, A. Biella, N. Bartolo, and C. Ciuti, Spectral theory of Liouvillians for dissipative phase transitions, Phys. Rev. A 98, 042118 (2018).

[63] P. Kirton and J. Keeling, Suppressing and Restoring the Dicke Superradiance Transition by Dephasing and Decay, Phys. Rev. Lett. 118, 123602 (2017).

[64] J. Keeling, M. J. Bhaseen, and B. D. Simons, Collective Dynamics of Bose-Einstein Condensates in Optical Cavities, Phys. Rev. Lett. 105, 043001 (2010).

[65] See Supplemental Material at http://link.aps.org/supplemental/ 10.1103/PhysRevResearch.3.L032016 for full expressions of the 4KRE, cumulant equations, and comparison of Liouvillian gap spacing to the Redfield theory of the Dicke model.

[66] G. Lindblad, On the generators of quantum dynamical semigroups, Commun. Math. Phys. 48, 119 (1976).

[67] J. Jeske, D. Ing, M. B. Plenio, S. F. Huelga, and J. H. Cole, Bloch-Redfield equations for modeling light-harvesting complexes, J. Chem. Phys. 142, 064104 (2015).

[68] P. R. Eastham, P. Kirton, H. M. Cammack, B. W. Lovett, and J. Keeling, Bath-induced coherence and the secular approximation, Phys. Rev. A 94, 012110 (2016).

[69] H. M. Cammack, P. Kirton, T. M. Stace, P. R. Eastham, J. Keeling, and B. W. Lovett, Coherence protection in coupled quantum systems, Phys. Rev. A 97, 022103 (2018).

[70] A. Dodin, T. Tscherbul, R. Alicki, A. Vutha, and P. Brumer, Secular versus nonsecular Redfield dynamics and Fano coherences in incoherent excitation: An experimental proposal, Phys. Rev. A 97, 013421 (2018).

[71] R. Hartmann and W. T. Strunz, Accuracy assessment of perturbative master equations: Embracing nonpositivity, Phys. Rev. A 101, 012103 (2020).

[72] R. Dümcke and H. Spohn, The proper form of the generator in the weak coupling limit, Z. Phys. B 34, 419 (1979).

[73] C. Gardiner, Stochastic Methods (Springer, Berlin, 2009).

[74] We label eigenvalues as $\lambda_{i}^{(k)}$, with $i=0,1,2, \ldots$. Hence, $\lambda_{0}^{(0)}=$ 0 is the zero mode associated to the steady state and $\lambda_{1}^{(0)}$ is the first nonzero eigenvalue in the $k=0$ sector.

[75] F. Minganti, I. I. Arkhipov, A. Miranowicz, and F. Nori, Liouvillian spectral collapse in Scully-Lamb lasing: Nonequilibrium second-order phase transition with or without $\mathrm{U}(1)$ symmetry breaking, arXiv:2103.05625.

[76] B. Buča and D. Jaksch, Dissipation Induced Nonstationarity in a Quantum Gas, Phys. Rev. Lett. 123, 260401 (2019).

[77] E. I. R. Chiacchio and A. Nunnenkamp, Dissipation-Induced Instabilities of a Spinor Bose-Einstein Condensate Inside an Optical Cavity, Phys. Rev. Lett. 122, 193605 (2019).

[78] R. Palacino and J. Keeling, 2020, dataset for "Data Underpinning: Atom-only theories for U(1) symmetric cavity-QED models", University of St Andrews Research Portal, 2021, doi: 10.17630/c780e316-128c-4f2c-84d0-f7ee7876938d. 